\title{
SYARAT CUKUP UNTUK OPTIMALITAS MASALAH KONTROL KUADRATIK LINIER
}

\author{
SUCI FRATAMA SARI \\ Program Studi Matematika, \\ Fakultas Matematika dan Ilmu Pengetahuan Alam, Universitas Andalas, \\ Kampus UNAND Limau Manis Padang, Indonesia, \\ sucifratamasari@rocketmail.com
}

\begin{abstract}
The LQR problem is an optimal control problem which is now used in various fields of science. The optimal control is given by $\mathbf{u}^{*}(t)=-K \mathbf{x}(t)$, where $K=R^{-1}(P B)^{T}$ and $P$ is a unique positive semidefinite solution of Algebraic Riccati Equation (ARE). The existence of optimal control $\mathbf{u}^{*}(t)$ depends on the existence matrix $P$. In this paper, the sufficient conditions which ensures the existence and uniqueness of the optimal control $\mathbf{u}^{*}(t)$ will be determined. Moreover, some examples as an illustration of the LQR problem will be given.
\end{abstract}

Kata Kunci: LQR, algebraic Riccati equation (ARE), stability.

\section{Pendahuluan}

Masalah kontrol kuadratik linier merupakan masalah penentuan suatu pengontrol optimal $\mathbf{u}^{*} \in \mathbb{R}^{m}$ yang meminimumkan fungsional

$$
J=\int_{0}^{\infty}\left[\mathbf{x}^{T} Q \mathbf{x}+\mathbf{u}^{T} R \mathbf{u}\right] d t
$$

di mana $\mathbf{u}^{*}$ memenuhi sistem dinamik

$$
\dot{\mathbf{x}}=A \mathbf{x}+B \mathbf{u}, \mathbf{x}(0)=\mathbf{x}_{0},
$$

dengan $Q$ adalah matriks simetris semidefinit positif, $R$ adalah matriks simetris definit positif, $A \in \mathbb{R}^{n \times n}, B \in \mathbb{R}^{n \times m}$ dan $t \geq 0$. Pada sistem (1.2), $\mathbf{x}=\mathbf{x}(t) \in \mathbb{R}^{n}$ menyatakan vektor keadaan (state), $\mathbf{u}=\mathbf{u}(t) \in \mathbb{R}^{m}$ menyatakan vektor kontrol (input) dan $t$ menyatakan waktu [6]. Notasi $\mathbb{R}^{n \times m}$ menyatakan himpunan matriks riil berukuran $n \times m$ dan $\mathbb{R}^{n}$ menyatakan himpunan vektor riil yang terdiri atas $n$ komponen.

Dalam Bolza [3], telah dibuktikan bahwa solusi optimal dari permasalahan tersebut adalah

$$
\mathbf{u}^{*}=-K \mathbf{x}
$$

di mana $K=R^{-1}(P B)^{T}$ dan $P$ adalah solusi definit positif dari persamaan aljabar Riccati

$$
A^{T} P+P A+Q-P B R^{-1}(P B)^{T}=0 .
$$


Persamaan (1.3) memperlihatkan bahwa eksistensi dari pengontrol optimal sangat bergantung kepada eksistensi matriks simetris $P$ yang memenuhi persamaan aljabar Riccati (1.4). Dalam skripsi ini, akan dikaji syarat cukup yang menjamin eksistensi dan ketunggalan pengontrol optimal tersebut untuk masalah kontrol kuadratik linier (1.1) dan (1.2).

\section{Syarat Cukup untuk Optimalitas Masalah Kontrol Kuadratik Linier}

Dalam bagian ini akan dijabarkan syarat cukup yang menjamin eksistensi dan ketunggalan pengontrol optimal $\mathbf{u}^{*}$ yang meminimumkan fungsional

$$
J=\int_{0}^{\infty}\left[\mathbf{x}^{T} Q \mathbf{x}+\mathbf{u}^{T} R \mathbf{u}\right] d t
$$

di mana $\mathbf{u}^{*}$ memenuhi sistem dinamik

$$
\dot{\mathbf{x}}=A \mathbf{x}+B \mathbf{u}, \mathbf{x}(0)=\mathbf{x}_{0},
$$

dengan $Q$ adalah matriks simetris semidefinit positif, $R$ adalah matriks simetris definit positif, $A \in \mathbb{R}^{n \times n}, B \in \mathbb{R}^{n \times m}$ dan $t \geq 0$.

Untuk mendapatkan syarat cukup tersebut, terlebih dahulu fungsional tujuan (2.1) diupayakan diubah dalam bentuk

$$
J=J_{0}+\int_{0}^{\infty}\left(\mathbf{u}-\mathbf{u}_{0}\right)^{T} R\left(\mathbf{u}-\mathbf{u}_{0}\right) d t
$$

di mana $J_{0}$ adalah suatu entitas yang bebas dari $\mathbf{u}$, dan $\mathbf{u}_{0}$ adalah suatu pengontrol baru yang akan dipilih. Jika ini dapat dilakukan, maka jelas bahwa minimum $J(\mathbf{u})$ dicapai pada $\mathbf{u}=\mathbf{u}_{0}, t \geq 0$, dengan nilai minimum adalah $J_{0}$. Permasalahan yang muncul adalah bagaimana bentuk eksplisit dari $J_{0}$ dan $\mathbf{u}_{0}$. Lema berikut diperlukan untuk mendapatkan pengontrol optimal $\mathbf{u}^{*}$.

Lema 3.1. [7] Misalkan $P \in \mathbb{R}^{n \times n}$ adalah suatu matriks simetris. Jika $\lim _{t \rightarrow \infty} \mathbf{x}(t)=\mathbf{0}$, untuk setiap kontrol $\mathbf{u}$ dengan $t \geq 0$, maka

$$
\int_{0}^{\infty}\left[\mathbf{x}^{T}\left(A^{T} P+P A\right) \mathbf{x}+2 \mathbf{x}^{T} P B \mathbf{u}\right] d t=-\mathbf{x}^{T}(0) P \mathbf{x}(0)
$$

\section{Bukti.}


$\int_{0}^{\infty}\left[\mathbf{x}^{T}\left(A^{T} P+P A\right) \mathbf{x}+2 \mathbf{x}^{T} P B \mathbf{u}\right] d t$

$$
\begin{aligned}
& =\int_{0}^{\infty}\left[\left(\mathbf{x}^{T} A^{T}+\mathbf{u}^{T} B^{T}\right) P \mathbf{x}+\mathbf{x}^{T} P(A \mathbf{x}+B \mathbf{u})\right] d t \\
& =\int_{0}^{\infty}\left(\dot{\mathbf{x}}^{T} P \mathbf{x}+\mathbf{x}^{T} P \dot{\mathbf{x}}\right) d t \\
& =\int_{0}^{\infty} \frac{d}{d t}\left(\mathbf{x}^{T} P \mathbf{x}\right) d t \\
& =\lim _{a \rightarrow \infty} \int_{0}^{a} \frac{d}{d t}\left(\mathbf{x}^{T} P \mathbf{x}\right) d t \\
& =\lim _{a \rightarrow \infty}\left(\left.\mathbf{x}^{T} P \mathbf{x}\right|_{0} ^{a}\right) \\
& =\lim _{a \rightarrow \infty}\left[\mathbf{x}^{T}(a) P \mathbf{x}(a)-\mathbf{x}^{T}(0) P \mathbf{x}(0)\right] \\
& =\lim _{t \rightarrow \infty}\left[\mathbf{x}^{T}(t) P \mathbf{x}(t)\right]-\mathbf{x}^{T}(0) P \mathbf{x}(0) \\
& =-\mathbf{x}^{T}(0) P \mathbf{x}(0) . \mathbf{0}
\end{aligned}
$$

Berdasarkan Lema (3.1), maka $J$ pada persamaan (2.1) dapat ditulis menjadi

$$
\begin{aligned}
J= & \int_{0}^{\infty}\left[\mathbf{x}^{T} Q \mathbf{x}+\mathbf{u}^{T} R \mathbf{u}\right] d t+\mathbf{x}^{T}(0) P \mathbf{x}(0) \\
& \left.+\int_{0}^{\infty}\left\{\mathbf{x}^{T}\left(A^{T} P+P A\right)\right) \mathbf{x}+2 \mathbf{x}^{T} P B \mathbf{u}\right\} d t \\
= & \mathbf{x}^{T}(0) P \mathbf{x}(0)+\int_{0}^{\infty}\left[\mathbf{x}^{T}\left(A^{T} P+P A+Q\right) \mathbf{x}+\mathbf{u}^{T} R \mathbf{u}\right. \\
& \left.+2 \mathbf{x}^{T} P B \mathbf{u}\right] d t .
\end{aligned}
$$

Selanjutnya, misalkan

$$
\mathbf{u}_{0}=-R^{-1}(P B)^{T} \mathbf{x}
$$

maka

$$
\begin{aligned}
\left(\mathbf{u}-\mathbf{u}_{0}\right)^{T} R\left(\mathbf{u}-\mathbf{u}_{0}\right)= & \left(\mathbf{u}+R^{-1}(P B)^{T} \mathbf{x}\right)^{T} R\left(\mathbf{u}+R^{-1}(P B)^{T} \mathbf{x}\right) \\
= & \mathbf{u}^{T} R \mathbf{u}+\mathbf{u}^{T}(P B)^{T} \mathbf{x}+\mathbf{x}^{T}(P B)\left(R^{-1}\right)^{T} R \mathbf{u} \\
& +\mathbf{x}^{T}(P B)\left(R^{-1}\right)^{T} R R^{-1}(P B)^{T} \mathbf{x} \\
= & \mathbf{u}^{T} R \mathbf{u}+\mathbf{u}^{T}(P B)^{T} \mathbf{x}+\mathbf{x}^{T}(P B)\left(R^{-1}\right)^{T} R^{T} \mathbf{u} \\
& +\mathbf{x}^{T}(P B)\left(R^{-1}\right)^{T} R^{T} R^{-1}(P B)^{T} \mathbf{x} \\
= & \mathbf{u}^{T} R \mathbf{u}+\mathbf{u}^{T}(P B)^{T} \mathbf{x}+\mathbf{x}^{T}(P B)\left(R R^{-1}\right)^{T} \mathbf{u} \\
& +\mathbf{x}^{T}(P B)\left(R R^{-1}\right)^{T} R^{-1}(P B)^{T} \mathbf{x} \\
= & \mathbf{u}^{T} R \mathbf{u}+\mathbf{u}^{T}(P B)^{T} \mathbf{x}+\mathbf{x}^{T}(P B) \mathbf{u} \\
& +\mathbf{x}^{T}(P B) R^{-1}(P B)^{T} \mathbf{x} \\
= & \mathbf{u}^{T} R \mathbf{u}+2 \mathbf{x}^{T} P B \mathbf{u}+\mathbf{x}^{T}(P B) R^{-1}(P B)^{T} \mathbf{x},
\end{aligned}
$$

atau dapat ditulis

$$
\mathbf{u}^{T} R \mathbf{u}+2 \mathbf{x}^{T} P B \mathbf{u}=\left(\mathbf{u}-\mathbf{u}_{0}\right)^{T} R\left(\mathbf{u}-\mathbf{u}_{0}\right)-\mathbf{x}^{T}(P B) R^{-1}(P B)^{T} \mathbf{x} .
$$


Dengan menggantikan (2.5) ke dalam (2.4), diperoleh

$$
J=J_{0}+\int_{0}^{\infty}\left(\mathbf{u}-\mathbf{u}_{0}\right)^{T} R\left(\mathbf{u}-\mathbf{u}_{0}\right) d t
$$

di mana

$$
J_{0}=\mathbf{x}^{T}(0) P \mathbf{x}(0)+\int_{0}^{\infty}\left[\mathbf{x}^{T}\left(A^{T} P+P A+Q-(P B) R^{-1}(P B)^{T}\right) \mathbf{x}\right] d t .
$$

Untuk menjamin agar $J_{0}$ bebas dari $\mathbf{u}$, pilih matriks simetris $P$ sedemikian sehingga

$$
A^{T} P+P A-P B R^{-1} B^{T} P+Q=0
$$

Jadi, pengontrol optimal untuk sistem (2.2) adalah

$$
\mathbf{u}^{*}=\mathbf{u}_{0}=-K \mathbf{x}, t \geq 0
$$

di mana $K=R^{-1}(P B)^{T}, P$ memenuhi persamaan aljabar Riccati $(2.6)$ dan $\mathbf{x}(t)$ memenuhi persamaan diferensial

$$
\dot{\mathbf{x}}=\left(A-B R^{-1}(P B)^{T}\right) \mathbf{x}, \quad \mathbf{x}(0)=\mathbf{x}_{0},
$$

dengan $J^{*}=\mathbf{x}^{T}(0) P \mathbf{x}(0)$.

Dengan memperhatikan lema (3.1) di mana $\lim _{t \rightarrow \infty} \mathbf{x}(t)=\mathbf{0}$ dan $\mathbf{x}$ memenuhi persamaan diferensial (2.8), maka ini bermakna bahwa sistem (2.2) dapat distabilkan.

Fakta (2.7) memperlihatkan bahwa eksistensi dari pengontrol optimal sangat bergantung kepada eksistensi matriks simetris $P$ yang merupakan solusi persamaan aljabar Riccati (2.6). Teorema berikut menjamin eksistensi $P$ tersebut.

Teorema 3.2. [7] Jika $(A, B)$ dapat distabilkan dan $\left(Q+K^{T} R K\right)$ adalah definit positif maka solusi definit positif persamaan aljabar Riccati (2.6) adalah tunggal, di mana diberikan oleh

$$
P=\int_{0}^{\infty} e^{(A-B K)^{T} t}\left(Q+K^{T} R K\right) e^{(A-B K) t} d t
$$

Bukti. Akan ditunjukkan bahwa $P$ adalah solusi dari (2.6). Persamaan (2.6) dapat diubah menjadi

$$
(A-B K)^{T} P+P(A-B K)=-\left(Q+K^{T} R K\right) .
$$

Subtitusikan (2.9) ke ruas kiri dari (2.10), diperoleh 


$$
\begin{aligned}
&(A-B K)^{T} P+P(A-B K) \\
&=\int_{0}^{\infty}(A-B K)^{T} e^{(A-B K)^{T} t}\left(Q+K^{T} R K\right) e^{(A-B K) t} d t \\
&+\int_{0}^{\infty} e^{(A-B K)^{T} t}\left(Q+K^{T} R K\right) e^{(A-B K) t}(A-B K) d t \\
&= \int_{0}^{\infty} \frac{d}{d t} e^{(A-B K)^{T} t}\left(Q+K^{T} R K\right) e^{(A-B K) t} d t \\
&= \lim _{a \rightarrow \infty} \int_{0}^{a} \frac{d}{d t} e^{(A-B K)^{T} t}\left(Q+K^{T} R K\right) e^{(A-B K) t} d t \\
&= \lim _{a \rightarrow \infty}\left(\left.e^{(A-B K)^{T} t}\left(Q+K^{T} R K\right) e^{(A-B K) t}\right|_{0} ^{a}\right) \\
&= \lim _{a \rightarrow \infty}\left[e^{(A-B K)^{T} a}\left(Q+K^{T} R K\right) e^{(A-B K) a}-e^{(A-B K)^{T} \cdot 0}\left(Q+K^{T} R K\right) e^{(A-B K) \cdot 0}\right] \\
&= \lim _{t \rightarrow \infty}\left[e^{(A-B K)^{T} t}\left(Q+K^{T} R K\right) e^{(A-B K) t}\right]-e^{(A-B K)^{T} \cdot 0}\left(Q+K^{T} R K\right) e^{(A-B K) .0} \\
&=-\left(Q+K^{T} R K\right) .
\end{aligned}
$$

Ini menunjukkan bahwa P merupakan solusi dari (2.6).

Selanjutnya akan ditunjukkan bahwa $P$ adalah definit positif. Dari (2.9) diperoleh

$$
\mathbf{x}^{T} P \mathbf{x}=\int_{0}^{\infty} \mathbf{x}^{T} e^{(A-B K)^{T} t}\left(Q+K^{T} R K\right) e^{(A-B K) t} \mathbf{x} d t, \text { untuk } \mathbf{x} \neq \mathbf{0} .
$$

Karena $Q+K^{T} R K$ adalah definit positif dan $e^{(A-B K) t} \mathbf{x} \neq \mathbf{0}, t \geq 0$, maka $\mathbf{x}^{T} P \mathbf{x}>$ 0 . Ini menunjukkan bahwa $P$ adalah definit positif.

Selanjutnya akan ditunjukkan bahwa $P$ adalah solusi tunggal. Misalkan $P_{n}$ adalah solusi lain dari (2.6), maka

$$
(A-B K)^{T} P_{n}+P_{n}(A-B K)=-\left(Q+K^{T} R K\right)
$$

dan

$$
(A-B K)^{T} P+P(A-B K)=-\left(Q+K^{T} R K\right) .
$$

Pengurangan (2.11) dengan (2.12) diperoleh

$$
(A-B K)^{T}\left(P_{n}-P\right)+\left(P_{n}-P\right)(A-B K)=0 .
$$

Selanjutnya,

$e^{(A-B K)^{T} t}(A-B K)^{T}\left(P_{n}-P\right) e^{(A-B K) t}+e^{(A-B K)^{T} t}\left(P_{n}-P\right)(A-B K) e^{(A-B K) t}=0$.

Persamaan (2.13) ekivalen dengan

$$
\frac{d}{d t} e^{(A-B K)^{T} t}\left(P_{n}-P\right) e^{(A-B K) t}=0, \quad t \geq 0 .
$$


Pengintegralan persamaan $(2.14)$ pada $[0, \infty)$ menghasilkan

$$
\begin{aligned}
& 0=\int_{0}^{\infty} \frac{d}{d t} e^{(A-B K)^{T} t}\left(P_{n}-P\right) e^{(A-B K) t} d t \\
& 0=\lim _{a \rightarrow \infty} \int_{0}^{a} \frac{d}{d t} e^{(A-B K)^{T} t}\left(P_{n}-P\right) e^{(A-B K) t} d t \\
& 0=\lim _{a \rightarrow \infty}\left(\left.e^{(A-B K)^{T} t}\left(P_{n}-P\right) e^{(A-B K) t}\right|_{0} ^{a}\right) \\
& 0=\lim _{a \rightarrow \infty}\left[e^{(A-B K)^{T} a}\left(P_{n}-P\right) e^{(A-B K) a}-e^{(A-B K)^{T} \cdot 0}\left(P_{n}-P\right) e^{(A-B K) .0}\right] \\
& 0=\lim _{t \rightarrow \infty}\left[e^{(A-B K)^{T} t}\left(P_{n}-P\right) e^{(A-B K) t}\right]-e^{(A-B K)^{T} \cdot 0}\left(P_{n}-P\right) e^{(A-B K) .0} \\
& 0=-\left(P_{n}-P\right) .
\end{aligned}
$$

Akibatnya $P_{n}=P$.

Dengan demikian, syarat cukup yang menjamin eksistensi dan ketunggalan pengontrol optimal untuk masalah kontrol kuadratik linier (2.1) dan (2.2) adalah $(A, B)$ dapat distabilkan dan matriks $Q+K^{T} R K$ adalah definit positif, di mana $K=R^{-1}(P B)^{T}$ dan $P$ adalah solusi persamaan aljabar Riccati (2.6).

Contoh berikut mengilustrasikan untuk permasalahan kontrol kuadratik linier.

\section{Contoh 1.}

$$
\begin{aligned}
& \min J=\int_{0}^{\infty}\left[2 x^{2}(t)+0.25 u^{2}(t)\right] d t \\
& \text { s.t. } \ddot{x}+x=u, x(0)=0, \dot{x}(0)=1
\end{aligned}
$$

Akan ditentukan kontrol optimal dari masalah (2.15).

Misalkan $\dot{x}=y$, maka $\dot{y}=-x+u$. Misalkan juga $P=\left(\begin{array}{ll}a & b \\ b & c\end{array}\right)$, maka permasalahan (2.15) dapat ditulis menjadi

$$
\begin{aligned}
\min & J=\int_{0}^{\infty}\left[\left(\begin{array}{ll}
x & y
\end{array}\right)\left(\begin{array}{ll}
2 & 0 \\
0 & 0
\end{array}\right)\left(\begin{array}{l}
x \\
y
\end{array}\right)+0,25 u^{2}\right] d t, \\
\text { s.t. } & \left(\begin{array}{l}
\dot{x} \\
\dot{y}
\end{array}\right)=\left(\begin{array}{cc}
0 & 1 \\
-1 & 0
\end{array}\right)\left(\begin{array}{l}
x \\
y
\end{array}\right)+\left(\begin{array}{l}
0 \\
1
\end{array}\right) u,
\end{aligned}
$$

dengan

$$
x(0)=0 \text { dan } y(0)=1 .
$$

Persamaan aljabar riccati untuk permasalahan (2.15) adalah

$$
\left(\begin{array}{cc}
-2 b+2-4 b^{2} & a-c-4 b c \\
a-c-4 b c & 2 b-4 c^{2}
\end{array}\right)=\left(\begin{array}{ll}
0 & 0 \\
0 & 0
\end{array}\right)
$$

atau ekivalen dengan

$$
\begin{array}{r}
-2 b+2-4 b^{2}=0 \\
a-c-4 b c=0 \\
2 b-4 c^{2}=0 .
\end{array}
$$


Solusi sistem persamaan non linier (2.17) adalah $a=1,5, b=0,5$, dan $c=0,5$. Jadi,

$$
P=\left(\begin{array}{ll}
1,5 & 0,5 \\
0,5 & 0,5
\end{array}\right)
$$

Sehingga, diperoleh

$$
\begin{aligned}
\mathbf{u}^{*} & =-R^{-1}(P B)^{T} \mathbf{x} \\
& =-2 x-2 y .
\end{aligned}
$$

Karena $\dot{y}=-x+u$, maka

$$
\dot{y}=-3 x-2 y .
$$

Karena $\dot{x}=y$, maka

$$
\begin{aligned}
\ddot{x} & =\dot{y} \\
& =-3 x-2 \dot{x} .
\end{aligned}
$$

Persamaan karakteristik dari persamaan differensial (2.18) adalah

$$
r^{2}+2 r+3=0,
$$

yang akar-akarnya adalah

$$
r_{1,2}=-1 \pm \sqrt{2} i .
$$

Sehingga solusi persamaan differensial (2.18) adalah

$$
\begin{aligned}
x(t) & =c_{1} e^{(-1+\sqrt{2} i) t}+c_{2} e^{(-1-\sqrt{2} i) t} \\
& =c_{1} e^{-t}(\cos \sqrt{2} t+i \sin \sqrt{2} t)+c_{2} e^{-t}(\cos \sqrt{2} t-i \sin \sqrt{2} t) \\
& =e^{-t}(C \cos \sqrt{2} t+D \sin \sqrt{2} t), \text { dengan } C=c_{1}+c_{2} \text { dan } D=\left(c_{1}-c_{2}\right) i .
\end{aligned}
$$

Dari (2.16) diperoleh

$$
C=0
$$

dan

$$
D=\frac{1}{\sqrt{2}}
$$

Jadi,

$$
\begin{aligned}
& x(t)=\frac{1}{\sqrt{2}} e^{-t} \sin \sqrt{2} t, \\
& y(t)=e^{-t} \cos \sqrt{2} t-\frac{1}{\sqrt{2}} e^{-t} \sin \sqrt{2} t .
\end{aligned}
$$

Sehingga, kontrol optimal untuk permasalahan (2.15) adalah

$$
\mathbf{u}^{*}(t)=-2 e^{-t} \cos \sqrt{2} t .
$$




\section{Kesimpulan}

Berdasarkan uraian dari pembahasan, maka pengontrol optimal $\mathbf{u}^{*}$ yang memininimumkan

$$
J=\int_{0}^{\infty}\left[\mathbf{x}^{T} Q \mathbf{x}+\mathbf{u}^{T} R \mathbf{u}\right] d t
$$

di mana $\mathbf{u}^{*}$ memenuhi sistem dinamik

$$
\dot{\mathbf{x}}=A \mathbf{x}+B \mathbf{u}, \mathbf{x}(0)=\mathbf{x}_{0},
$$

dengan dengan $Q$ adalah matriks simetris semidefinit positif, $R$ adalah matriks simetris definit positif, $A \in \mathbb{R}^{n \times n}, B \in \mathbb{R}^{n \times m}$ dan $t \geq 0$. adalah

$$
\mathbf{u}^{*}=-K \mathbf{x}, t \geq 0 .
$$

Pada sistem (3.2), $\mathbf{x}=\mathbf{x}(t) \in \mathbb{R}^{n}$ menyatakan vektor keadaan (state), $\mathbf{u}=\mathbf{u}(t) \in$ $\mathbb{R}^{m}$ menyatakan vektor kontrol (input) dan $t$ menyatakan waktu.

Syarat cukup untuk eksistensi dan ketunggalan kontrol optimal tersebut adalah $(A, B)$ dapat distabilkan dan matriks $Q+K^{T} R K$ adalah definit positif, di mana $K=R^{-1}(P B)^{T}$ dan $P$ adalah solusi yang memenuhi persamaan aljabar Riccati

$$
A^{T} P+P A-P B R^{-1} B^{T} P+Q=0 .
$$

\section{Ucapan Terima kasih}

Penulis mengucapkan terima kasih kepada Bapak Muhafzan, Ibu Arrival Rince Putri, Bapak Admi Nazra, Ibu Lyra Yulianti dan Ibu Nova Noliza Bakar yang telah memberikan masukan dan saran sehingga paper ini dapat diselesaikan dengan baik.

\section{Daftar Pustaka}

[1] Anton, H. dan C. Rorres. 2004. Aljabar Linear Elementer Edisi Kedelapan. Erlangga, Jakarta.

[2] Antsaklis, Panos J. and Anthony N. Michel. 2007. A Linear Systems Primer . Birkhauser, Boston.

[3] Bolza, O. 1904. Lectures on The Calculus of Variations. The Decennial Publications, Chicago.

[4] Meyer, C.D. 2000. Matrix Analysis and Applied Linier Algebra. SIAM, New York.

[5] Naidu, D.S. 2002. Optimal Control Systems. CRC Press, Idaho.

[6] Ogata, K. 2002. Modern Control Engineering. Aeeizh, Iran.

[7] Rugh, W.J. 1996. Linear System Theory. Prentice Hall, USA. 